\title{
iTutor: a Wireless and Multimodal Support to Industrial Maintenance Activities
}

\author{
Luca Tummolini, Andrea Lorenzon, Giancarlo Bo, and Roberto Vaccaro \\ Giunti Interactive Labs S.r.l., \\ Via Portobello, Abbazia dell'Annunziata, 16039 Sestri Levante, Italy \\ \{1.tummolini,a.lorenzon,g.bo,r.vaccaro\}@giuntilabs.it \\ http://www.giuntilabs.com
}

\begin{abstract}
This paper is focused on the description of the iTutor application. iTutor addresses the increasing needs originating in contemporary industrial workspaces for the delivery of electronic information within learning, knowledge transfer and information management processes. In order to achieve the goal of a flexible and adaptable information delivery in the working environment, iTutor integrates a mobile wearable device with a voice and gaze controlled, web-based graphical user interface based on the XML standard. The application is wirelessly integrated with the enterprise learning \& information system where the technical information and course are stored. iTutor is meant to support the working activity of maintainers needing to have hands free in order to complete their tasks.
\end{abstract}

\section{Introduction}

Mobile services support the emerging increasing need of leisure as well as of working sectors to receive information and training anytime and anywhere.

More specifically, in the industrial maintenance sector, the organization of productive plants requires teams of spatially distributed working people. In order to improve productivity, these groups need to retrieve information as quickly as possible to complete repairing tasks. Moreover, due to downsizing of personnel, new ways of improving learning tasks are needed to complement the activity of experts providing on-line courses. Wearable and Mobile computers combined with wireless technology have been widely adopted to meet these general user requirements, [1], [2].

While mobility and remote access to enterprise information systems are generally pursued, only few applications have considered that traditional human-computer interaction modalities strongly impede the repairing task.

The general goal of iTutor is to support and ease maintenance activity and to provide professional training in an industrial setting where a user must keep his/her hands free to work with the target of intervention. To this end it has been designed and implemented in order to run on a wearable device (Xybernaut MAIV) that is wirelessly integrated with the industrial information system. The user can browse multimedia technical information (drawings, schemas, procedures and manuals) in a multimodal way, by simply using eye movements and speech commands. This way, 
iTutor offers a seamless mobile integration of information processing tools with the existing working environment.

The role of gaze based interaction with the system has been deeply investigated and a prototype providing information as a response to natural eye fixations has been developed and integrated.

In the following sections the main features and the underlying architecture of the system will be described. A summary of the results obtained during a preliminary usability evaluation campaign will be provided too.

\section{2 iTutor: a gaze and speech controlled application}

Besides the challenges faced during the design of the architecture of such system, the novelty and originality of the system mainly resides in the Graphical User Interface and the modalities of interaction with it. Essentially, the iTutor User Interface is a web-based application that allows the user to remotely navigate the information contained in the enterprise information system by exploiting a common Internet browser (Fig.1).
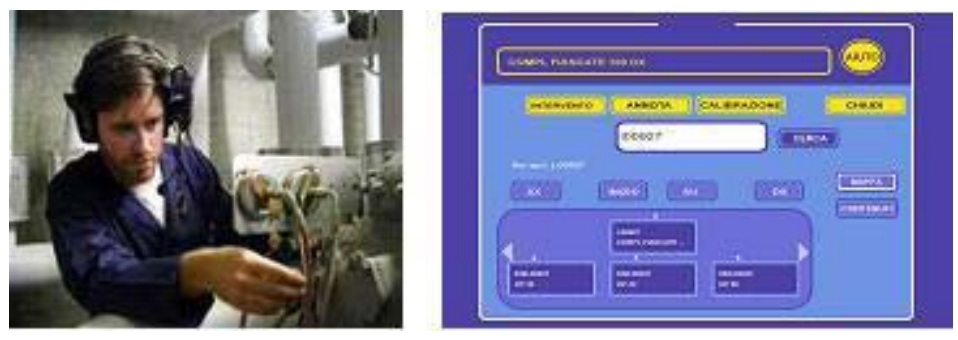

Fig. 1. Main iTutor Graphical User Interface

The complete structure of an industrial plant is represented by a large amount of information usually hierarchically organized. In iTutor a plant is represented by the Map Area: maintainers can browse through the different levels of a plant structure simply by "looking" at the different items in order to select them. Each rectangle in the map represents a different level in the actual plant structure (e.g. lines, machines, groups and elements). Through voice commands, a user can access specific information and assets related to a selected component (e.g..its position in the warehouse, available manuals and procedures, mechanical and electrical schemas, etc.). Speech input is acquired and managed by the multilingual Voice Recognition software, provided by partner Conexor (Finland), which has been integrated in the application.

The concept of interconnected schemas has been introduced in iTutor to ease the specific task of electronic troubleshooting. In order to let the user access the same detail/component in different representations (electrical schema, block diagram, serigraphy) the user can select the element of interest through eye fixations (Fig. 2). 


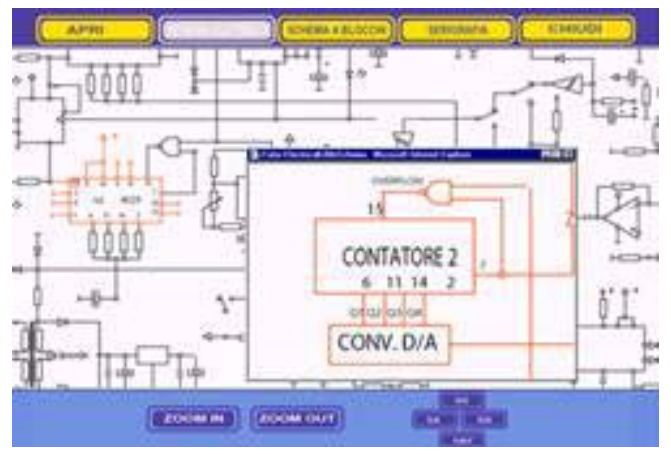

Fig. 2. Electrical schemas navigation page

The Intention Extraction Module (IEM) represents the core of the eye-based interaction. It functions as an interpreter of the user's fixations, which are acquired by a small USB eye tracker (the EyeMouse, produced by SensoMotoric Instruments, Germany), with respect to the active portions of the graphical interface. The problem of locating a gaze point on the user interface has been treated as a classification process (adopting a K-NN algorithm, see [3]) while the decision space is represented by an R-Tree, [4]. The IEM guarantees the direct interpretation of the user's intentions from the statistics of the eye gaze, enabling a noncommand style of interaction: the computer senses what the user wants to do without the user having to give any explicit command, [5].

From the point of view of the overall architecture, iTutor has been conceived and designed as a Web-based client-server application (Fig. 3).

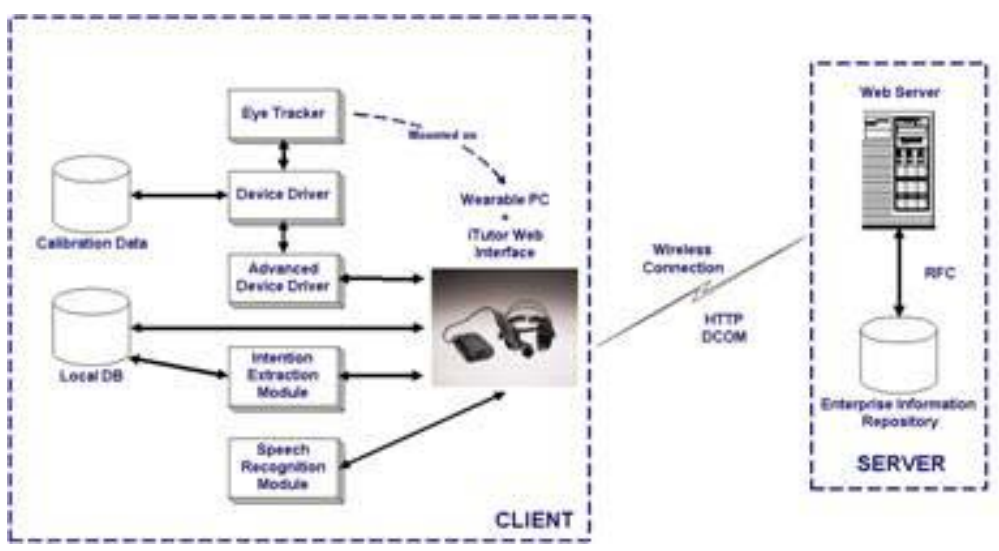

Fig. 3. Architecture of iTutor: a Web-based client-server application

On the Client Side three main components of the architecture can be located: the Eye Tracking Device and the related Advanced Device Driver, the Intention Extraction Module and the Speech Recognition Module. The Server Side of the 
application is organized as a two-layer server architecture: a Web Server is mainly devoted to the generation of the ASP pages that set iTutor's interface, while an Internal Server supports the communication with the DBMS (Database Management System) of the Enterprise Information System and with Europe's leading Scorm Learning Content Authoring \& Management Suite Learn eXact (www.learnexact.com) provided by Giunti Interactive Labs.

Finally, the integration in iTutor of an IEEE $802.11 \mathrm{~b}$ compliant WLAN allows maintainers to freely move through the plant, while working, without loosing contact with the source of the needed information.

\section{Conclusions}

A mobile and multimodal computer system to support maintenance and repair processes was designed and realized. iTutor was developed in collaboration with COMAU Service (FIAT group) which provided also the industrial site for scenario based evaluation with real users (automotive plant maintainers). The positive feedback obtained clearly underlines the system effectiveness fulfilling the main users requirements. Some modifications to the interface will be necessary for its improvement and specific functionalities will be added to the system.

\section{Acknowledgments}

This work has been undertaken in the framework of the iEye Project (IST-199911883) which is partly funded by the European Commission under the Information Society Technologies 5th Framework Programme. The Authors would like to acknowledge the contributions of their colleagues from University of Tampere, Conexor, SensoMotoric Instruments and University of Nottingham.

\section{References}

1. Siewiorek, D., Smailagic, A., Bass, L., Siegel, J., Martin, R., Bennington, B.: Adtranz: A Mobile Computing System for Maintenance and Collaboration. In: Proc. IEEE International Conference on Wearable Computers, Pittsburgh, PA, October 1998.

2. Ockerman, J. J., Najjar, L. J., \& Thompson, J. C.: Factory automation support technology (FAST). In D. Adelson \& E. Domeshek (Eds.) International Conference on the Learning Sciences, 1996 (p. 567). Charlottesville, VA: Association for the Advancement of Computing in Education.

3. Roussopoulos, N., Kelley, S., Vincent, F.: Nearest neighbor queries. In: Proceedings of the 1995 ACMSIGMOD Intl.Conf. on Management of Data, San Jose, CA, June 1995.

4. Guttman, A.: R-trees: A dynamic index structure for spatial searching. In: Proc. ACM SIGMOD Int. Conf. on Management of Data, pages $45-57,1984$

5. Nielsen, J.: Non-command user interfaces. In: Communications of the ACM, 36(4), 1994, 83-99. 